\title{
Optimization of fatty alcohol biosynthesis pathway for selectively enhanced production of C12/14 and C16/18 fatty alcohols in engineered Escherichia coli
}

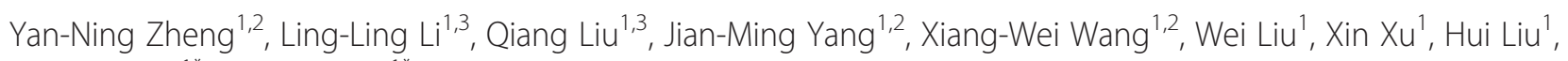
Guang Zhao ${ }^{1^{*}}$ and Mo Xian ${ }^{1 *}$

\begin{abstract}
Background: With the increasing stress from oil price and environmental pollution, aroused attention has been paid to the microbial production of chemicals from renewable sources. The C12/14 and C16/18 alcohols are important feedstocks for the production of surfactants and detergents, which are widely used in the most respected consumer detergents, cleaning products and personal care products worldwide. Though bioproduction of fatty alcohols has been carried out in engineered E. coli, several key problems have not been solved in earlier studies, such as the quite low production of C16/18 alcohol, the lack of optimization of the fatty alcohol biosynthesis pathway, and the uncharacterized performance of the engineered strains in scaled-up system.

Results: We improved the fatty alcohol production by systematically optimizing the fatty alcohol biosynthesis pathway, mainly targeting three key steps from fatty acyl-acyl carrier proteins (ACPs) to fatty alcohols, which are sequentially catalyzed by thioesterase, acyl-coenzyme A (CoA) synthase and fatty acyl-CoA reductase. By coexpression of thioesterase gene BTE, acyl-CoA synthase gene fadD and fatty acyl-CoA reductase gene acr1, $210.1 \mathrm{mg} / \mathrm{L} \mathrm{C12/14}$ alcohol was obtained. A further optimization of expression level of BTE, fadD and acr increased the $\mathrm{C} 12 / 14$ alcohol production to $449.2 \mathrm{mg} / \mathrm{L}$, accounting for $75.0 \%$ of the total fatty alcohol production $(598.6 \mathrm{mg} / \mathrm{L})$. In addition, by coexpression of thioesterase gene 'tes $A$, acyl-CoA synthase gene fadD and fatty acyl-CoA reductase gene FAR, $101.5 \mathrm{mg} / \mathrm{L}$ C16/18 alcohol was obtained, with C16/18 alcohol accounting for 89.2\% of the total fatty alcohol production.

Conclusions: To our knowledge, this is the first report on selective production of C12/14 and C16/18 alcohols by microbial fermentation. This work achieved high-specificity production of both C12/14 and C16/18 alcohols. The encouraging $598.6 \mathrm{mg} / \mathrm{L}$ of fatty alcohols represents the highest titer reported so far. In addition, the $101.5 \mathrm{mg} / \mathrm{L}$ 89.2\% C16/18 alcohol suggests an important breakthrough in C16/18 alcohol production. A more detailed optimization of the expression level of fatty alcohol biosynthesis pathway may contribute to a further improvement of fatty alcohol production.
\end{abstract}

Keywords: Fatty alcohol, Escherichia coli, Pathway optimization, Selective production, Fermentation

\footnotetext{
*Correspondence: zhaoguang@qibebt.ac.cn; xianmo@qibebt.ac.cn

${ }^{1}$ Qingdao Institute of Bioenergy and Bioprocess Technology, Chinese Academy of Sciences, No.189 Songling Road, Qingdao Laoshan District 266101, China

Full list of author information is available at the end of the article
} 


\section{Background}

Fatty alcohols, usually with hydrocarbon chains ranging from C8 to C18, are composed of a nonpolar, lipophilic carbon chain and a polar, hydrophilic hydroxyl group. The C8/10 alcohol (a blend of C8 and C10 alcohols) only has limited applications in the production of plasticizers, while the $\mathrm{C} 12-\mathrm{C} 18$ alcohols find applications in a multitude of uses. The C12/14 alcohol (a blend of C12 and $\mathrm{C} 14$ alcohols) can be used as lubricant additives, and the C16/18 alcohol (a blend of C16 and C18 alcohols) can be employed as defoamers, solubility retarders, and consistency giving factors. More importantly, the C12-C18 alcohols can be used to produce detergents and surfactants by replacing their hydroxy group with other larger hydrophilic groups $[1,2]$. Now the fatty alcohol market is dominated by natural alcohol and synthetic alcohol products. The natural alcohols are prepared from natural oil such as coconut oil and palm oil using transesterification and hydrogenation processes. The synthetic alcohols are produced from petrochemical feedstocks mainly using Ziegler-Process and OxoProcess. However, these processes either need harsh production environments, or bring harmful materials to the environment. Besides, they are both now facing the huge challenge from the increasingly higher price of the raw materials. Therefore, increasing attentions have been paid to the microbial production of fatty alcohols from renewable resources $[3,4]$.

The biosynthesis of fatty alcohols mainly employs the de novo fatty acid synthetic pathway of microbes. Fatty acyl-acyl carrier proteins (ACPs) are firstly manufactured via the fatty acid biosynthesis pathway. Then fatty acylACPs are converted to free fatty acids (FFAs) and fatty acyl-coenzyme A (CoA) sequentially catalyzed by thioesterase and acyl-CoA synthase [5]. Next, the fatty acylCoAs are reduced to fatty aldehydes or fatty alcohols in a NADPH-dependent reaction catalyzed by fatty acylCoA reductase. The synthesized fatty aldehydes can be further converted to fatty alcohols by an unknown alcohol dehydrogenase or aldehyde reductase of E. coli (Figure 1) [6,7].

The thioesterase, acyl-CoA synthase and fatty acylCoA reductase play the key role in the fatty alcohol biosynthesis [3]. Either thioesterases, acyl-CoA synthases or fatty acyl-CoA reductases from different organisms have differences in substrate specificity [8]. For examples, the thioesterase BTE from Umbellularia californica prefers the medium-chain acyl-ACPs [9-11], and the thioesterase 'TesA (a 'leaderless' version of TesA) from $E$. coli possesses a much broader substrate preference $[3,5,12]$. As for the fatty acyl-CoA synthase, the FadD from E. coli can efficiently activate the fatty acids ranging from $\mathrm{C} 10$ to $\mathrm{C} 18$, but FAA2 from Saccharomyces cerevisiae prefers the medium-chain fatty

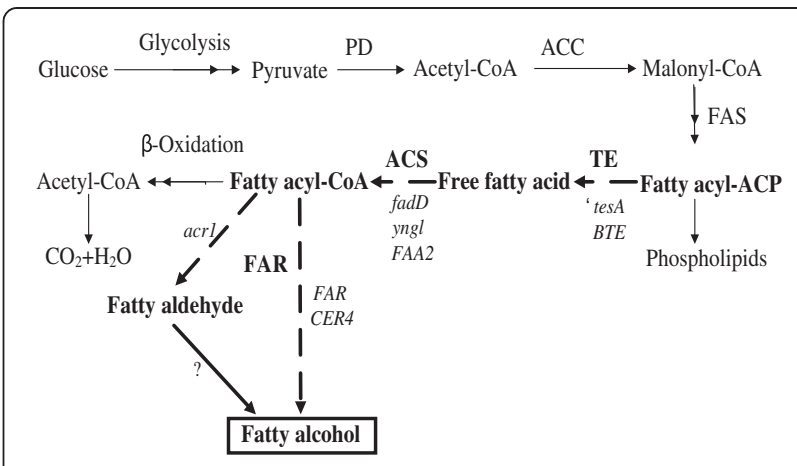

Figure 1 Biosynthetic pathways of fatty alcohol. The nodes we selected for metabolic engineering are marked in bold. The real lines represent the native pathways of $E$. coli, while the broken line shows a nonnative pathway of the host. The actual genes used for each step are shown in italics. PD: pyruvate dehydrogenase; ACC: acetylCoA carboxylase; FAS: fatty acid synthase; ACP: acyl carrier protein; TE: thioesterase ('tesA and BTE); ACS: acyl-CoA synthase (fadD, yngl and FAA2); FAR: fatty acyl-COA reductase (FAR, acr1 and CER4).

acids $[13,14]$. Many fatty acyl-CoA reductases, such as Acr1, FAR and CER4, are capable of converting fatty acyl-CoAs to fatty aldehydes (Acr1) or fatty alcohols (FAR and CER4) [6,15-18]. To investigate the substrate specificities of FAR and CER4, Doan et al. determined the composition of the fatty alcohols obtained by expressing a single FAR or CER4 in E. coli [19]. It was not a compellent strategy for evaluating the substrate specificity of fatty acyl-CoA reductase, since the FFAs with different chain lengths are not equally accumulated in the host. Though the substrate specificity of Acr1 was characterized in vitro by Reiser and Somervill [6], it is also needed to further characterize its substrate specificity in vivo to better simulate the cytocatalytic production of fatty alcohols.

Based on the achieved knowledge of the fatty alcohol biosynthesis pathway, microbial production of fatty alcohols was carried out in engineered E. coli [3]. Steen et al. enhanced the production of the medium chain fatty alcohols in an engineered $E$. coli by overexpression of the fatty alcohol biosynthesis pathway and deletion of the endogenous $f a d E$ gene (encoding acyl-CoA dehydrogenase) of E. coli. In addition, they made attempts to tailor the composition of fatty alcohols by employing thioesterases preferring different chain-length fatty acylACPs. The C12 alcohol was mainly produced by expressing UcFatB, while expression of ChFatB3 or 'tesA mainly contributed to production of $\mathrm{C} 14$ alcohol. However, only a small proportion of C16/18 alcohol was observed in their engineered strains [3].

Though fatty alcohols have been produced by engineered E. coli, however, several problems have not been solved in previous studies. First, no improvement has been achieved in the production of C16/18 alcohol; 
Second, detailed optimization of the fatty alcohol biosynthesis pathway has not been carried out; Third, the performance of the engineered $E$. coli on the production of fatty alcohols has not been investigated in scaleable fermentation processes.

In order to optimize the fatty alcohol biosynthesis pathway for selectively enhanced production of C12/14 and C16/18 alcohols, we investigated the effect of acylCoA synthases and fatty acyl-CoA reductases on the production of fatty alcohols, determined the C12/14 and C16/18 alcohol production across different thioesterases, acyl-CoA synthases and fatty acyl-CoA reductases, optimized the expression level of associated enzymes, and evaluated the biocatalytic properties of the engineered strains in fed-batch fermentation.

\section{Results}

Fatty alcohol distribution in engineered strains employing fatty acyl-CoA reductases FAR, Acr1 or CER4

To employ the proper fatty acyl-CoA reductases for selective production of $\mathrm{C} 12 / 14$ and $\mathrm{C} 16 / 18$ alcohols, we determined the fatty alcohol distribution of the engineered strains Zh05, Zh07 and Zh08 by adding an equivalent blend of lauric acid (C12:0), myristic acid (C14:0), palmitic acid (C16:0), stearic acid (C18:0) and oleic acid (C18:1) (200 mg/L each) into their cultures. Zh05, Zh07, and Zh08 overexpressed FAR gene from $S$. chinensis, acr1 gene from A. baylyi and CER4 gene from A. thaliana, respectively. To promote the conversion of FFAs to fatty acyl-CoAs, an endogenous acyl-CoA synthase gene $(f a d D)$ was also coexpressed in all three engineered strains. Given FadD has close substrate specificities towards C10 to C18 FFAs [13], the substrate preferences of CER4 and FAR can be roughly speculated according to the chain length distributions of the synthesized fatty alcohols. Though the substrate specificity of Acr1 can not be concluded from the distribution of fatty alcohols, the combined effect of Acr1 and endogenous alcohol dehydrogenase/aldehyde reductase on the fatty alcohol production can be clearly determined with the expression of Acr1 in E. coli. In addition, the endogenous FFAs of the host were determined in the same conditions where FFAs are supplied in the culture. The host E. coli BL21(DE3) only produced $15.7 \mathrm{mg} / \mathrm{L}$ FFAs (C12, $1.1 \mathrm{mg} / \mathrm{L} ; \mathrm{C} 14,2.0 \mathrm{mg} / \mathrm{L} ; \mathrm{C} 16,7.2 \mathrm{mg} / \mathrm{L}$; $\mathrm{C} 18,5.4 \mathrm{mg} / \mathrm{L})$, and the engineered strains Zh05, Zh07 and Zh08 produced nearly the same endogenous FFAs. Therefore, the endogenous FFAs of the hosts can be almost neglected when compared with fatty acids exogenously supplemented.

Fatty alcohol production in these engineered strains was analyzed by GC-MS. Zh07 and Zh08 produced a predominant C12/14 alcohol, 85.8\% and 97.7\%, respectively, with nearly no C18 alcohol produced, while Zh05 produced $81.1 \% \mathrm{C} 16 / 18$ alcohol, with C18 alcohol accounting for $75.9 \%$ (Figure 2). That is to say, when combined the expression of FadD with Acr1 or CER4, the engineered strains produced a predominant C12/14 alcohol, and when combined the expression of FadD with FAR, the engineered strain produced a predominant C16/18 alcohol. It demonstrates that CER4 prefers acyl group with relatively shorter carbon chain lengths, while FAR prefers a longer acyl group, especially the oleoyl group. The expression of Acr1 contributed to the synthesis of C12/14 alcohol, with the help of endogenous alcohol dehydrogenase/aldehyde reductase. It was worth mentioning that Zh05, Zh07 and Zh08 produced neglectable stearyl alcohol, and the C18 alcohol produced was predominately oleyl alcohol. In addition, Zh07 produced 39.6-fold more C12/14 alcohol than Zh08, suggesting expression of Acr 1 in E. coli is capable of producing more $\mathrm{C} 12 / 14$ alcohol than expression of CER4.

\section{Comparison of the efficiencies of three different acyl-CoA} synthases on fatty alcohol production

As above mentioned, the desired fatty acyl-CoA reductases have been obtained. And the substrate specificities of thioesterases BTE and 'TesA have been well characterized. BTE produces a predominant C12/14 FFAs, and 'TesA mainly produces C14-C18 FFAs. To better activate the specific FFAs to corresponding fatty acyl-CoAs, it is necessary to overexpress a proper acyl-CoA synthase. Therefore, we compared the efficiencies of three different

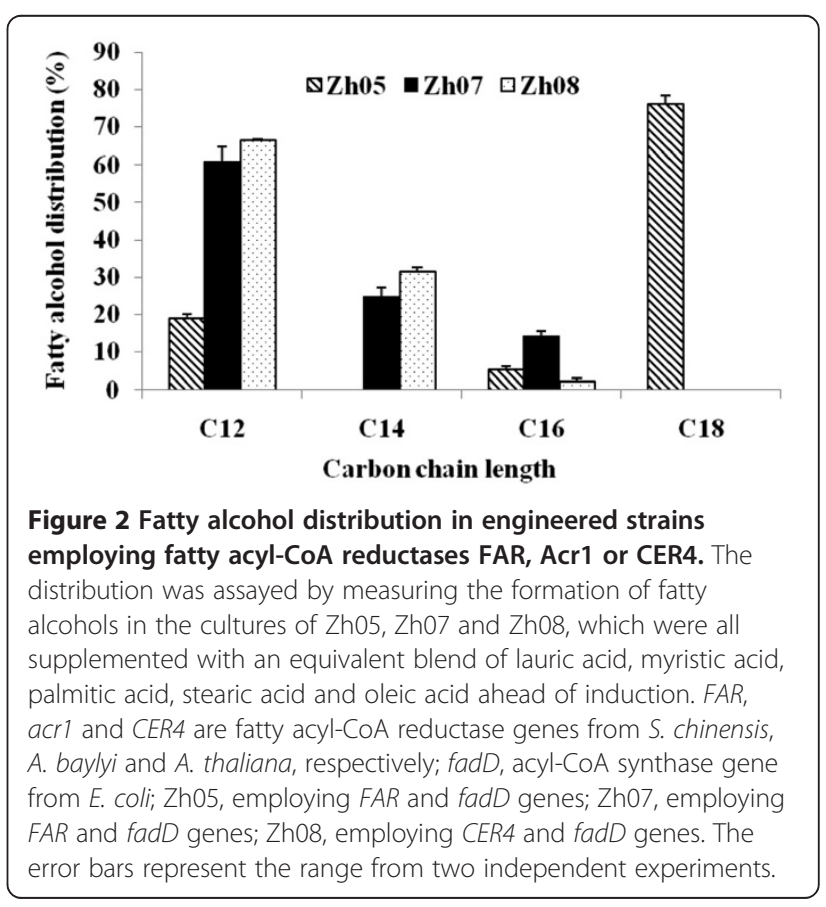


acyl-CoA synthases (FadD, Yngl and FAA2) on the production of C12/14 and C16/18 alcohols from glucose.

When coexpressed with BTE and Acr1, the strain with FadD overexpressed (Zh072) produced $13.3 \mathrm{mg} / \mathrm{L} \mathrm{C12/14}$ alcohol in shake flask, 23.6-fold and 16.8-fold higher than the strains employing Yngl from B. subtilis (Zh112) and FAA2 (Zh122) from S. cerevisiae, respectively (Figure $3 \mathrm{~A}$ ). In addition, Zh072 produced C12/14 alcohol as its major fatty alcohol constituent (78.6\%), with C10 alcohol (9.3\%), C16 alcohol (5.2\%) and $\mathrm{C} 18$ alcohol (6.9\%) being the other fatty alcohols observed (Figure 4).

To further confirm that acyl-CoA synthases contributed to the difference in the production of $\mathrm{C} 12 / 14$ alcohol, we determined the FFAs unutilized by these engineered strains. In contrast to $52.7 \mathrm{mg} / \mathrm{L}$ and $41.3 \mathrm{mg} / \mathrm{L} \mathrm{C12/14}$ acid unutilized by Zh112 and Zh122, respectively, only $2.1 \mathrm{mg} / \mathrm{L} \mathrm{C12/14}$ acid was not utilized by Zh072 (Figure 3B). It demonstrated that FadD could activate $\mathrm{C} 12 / 14$ acid to $\mathrm{C} 12 / 14$ acyl-CoA much more efficiently than Yngl and FAA2, and the higher reaction rate of FadD contributed to Zh072 achieving a higher C12/14 alcohol titer than Zh112 and Zh122.

We then compared their efficiencies on the production of C16/18 alcohol. When coexpressed with 'TesA and FAR, the strain with FadD overproduction (Zh054) produced $2.45 \mathrm{mg} / \mathrm{L} \mathrm{C16/18}$ alcohol in shake flask, 4.95fold and 4.44-fold higher than that of the strains employing Yngl (Zh094) and FAA2 (Zh104), respectively (Figure 3C). This result illuminated that, compared with Yngl and FAA2, FadD could direct more C16/18 acid to corresponding acyl-CoA, which was further reduced to C16/18 alcohol by FAR. This conclusion could be further confirmed by the analysis of the FFAs unutilized. Only $8.3 \mathrm{mg} / \mathrm{L}$ C16/18 acid was unutilized by Zh054, in contrast to $29.5 \mathrm{mg} / \mathrm{L}$ and $33.1 \mathrm{mg} / \mathrm{L} \mathrm{C16/18} \mathrm{acid} \mathrm{unuti-}$ lized by Zh094 and Zh104, respectively (Figure 3D).

We also analyzed the composition of fatty alcohols synthesized by Zh054. Zh054 produced C16/18 alcohol as its predominant fatty alcohol constituent (89.2\%),
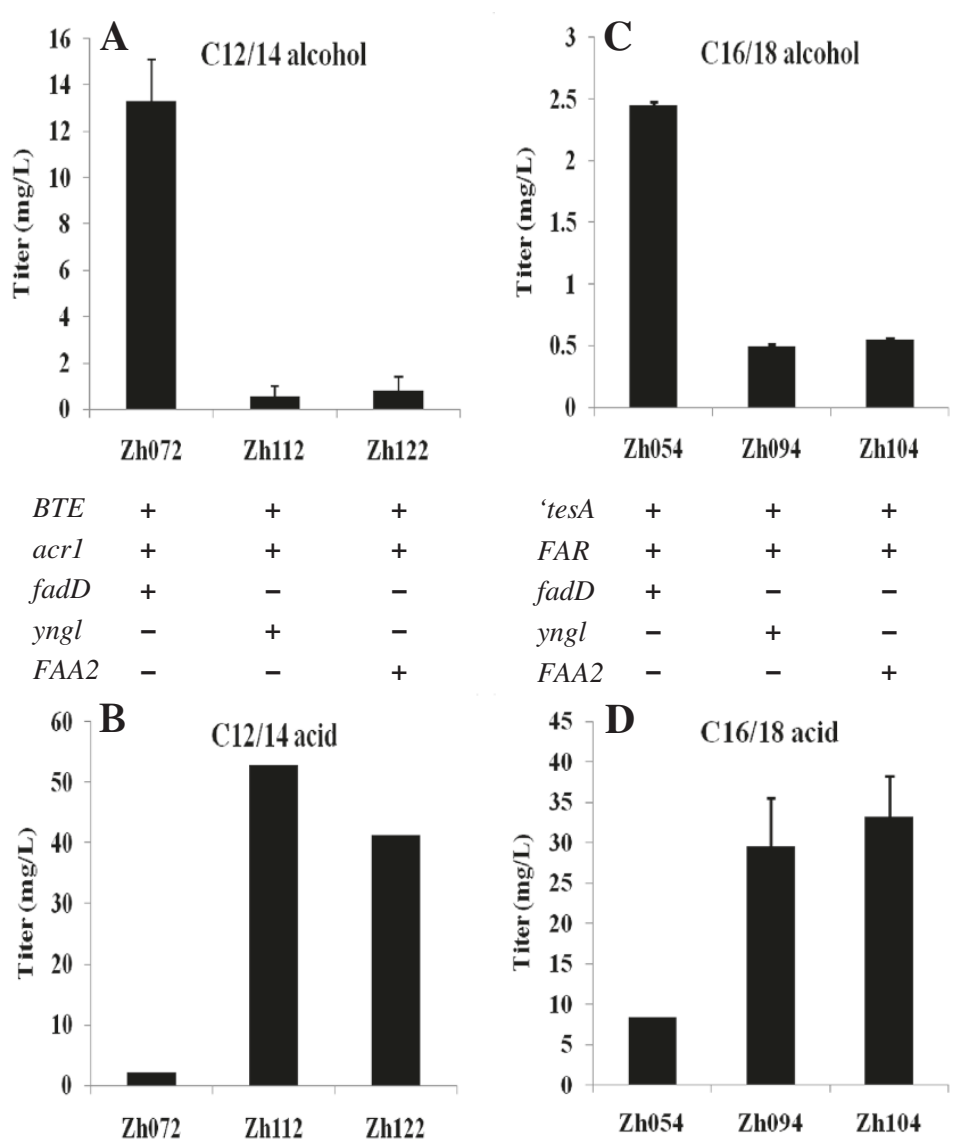

Figure 3 The efficiencies of FadD, Yngl and FAA2 on the production of fatty alcohols and the activation of FFAs. FadD, Yngl and FAA2 are acyl-CoA synthases from E. coli, B. subtilis and S. cerevisiae, respectively. A, B, the C12/14 alcohol production (A) and the C12/14 acid unutilized (B) by employing FadD, Yngl or FAA2 as the acyl-CoA synthase. C, D, the C16/18 alcohol production (C) and the C16/18 acid unutilized (D) by employing FadD, Yngl or FAA2 as the acyl-CoA synthase. The error bars represent the range from two independent experiments. 


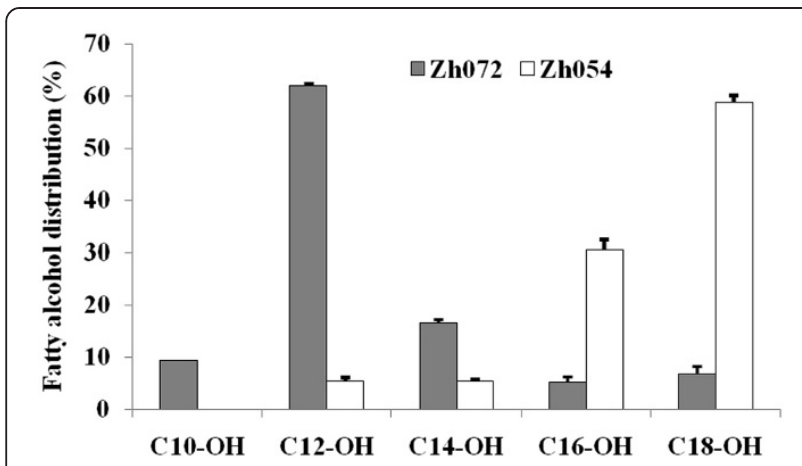

Figure 4 Fatty alcohol composition of Zh072 and Zh054 cultures in shake flask. Filled columns are for Zh072 (coexpression of Acr1, FadD and BTE); Open columns are for Zh054 (coexpression of FAR, FadD and TesA). The error bars represent the range from two independent experiments.

with $\mathrm{C} 12$ alcohol (5.4\%) and C14 alcohol (5.4\%) being the other fatty alcohols observed (Figure 4).

\section{Optimization of the expression level of $\mathrm{C} 12 / 14$ alcohol biosynthesis pathway}

Thus far, we have obtained two engineered strains Zh072 and Zh054, which were well performed in high-specificity production of $\mathrm{C} 12 / 14$ alcohols and $\mathrm{C} 16 / 18$ alcohols, respectively. However, it is possible to further enhance the production of fatty alcohols in E. coli by optimizing the expression level of fatty alcohol biosynthesis pathway. Given C12/14 alcohol and C16/18 alcohol biosynthesis pathways are quite similar to each other, we only did the optimization of the expression level of C12/14 alcohol biosynthesis pathway.

Firstly, several plasmids with different copy numbers were employed to bear BTE and fadD-acr1. When $B T E$ and $f a d D$-acr 1 were carried by a higher copy number plasmid and a lower copy number plasmid, respectively, corresponding strain Zh172 achieved the highest C12/14 alcohol titer. Zh072 using two higher copy number plasmids accumulated a 2.6-fold lower titer than Zh172. Zh0712 and Zh18, both carrying a lower copy number BTE, produced much lower $\mathrm{C} 12 / 14$ alcohol titers when compared with Zh072 and Zh172 (Figure 5).

The genes above mentioned are all under the control of the strong T7 promoter. Given Lennen et al. achieved a high production of fatty acids by using a medium-strength araBAD promoter $\left(\mathrm{P}_{\mathrm{BAD}}\right)[20]$, we thus partly replace the T7 promoter with $\mathrm{P}_{\mathrm{BAD}}$. But unfortunately, none of the engineered strains improved the production of $\mathrm{C} 12 / 14$ alcohol when either BTE or acrl gene was under the control of $\mathrm{P}_{\mathrm{BAD}}$ (Figure 5).

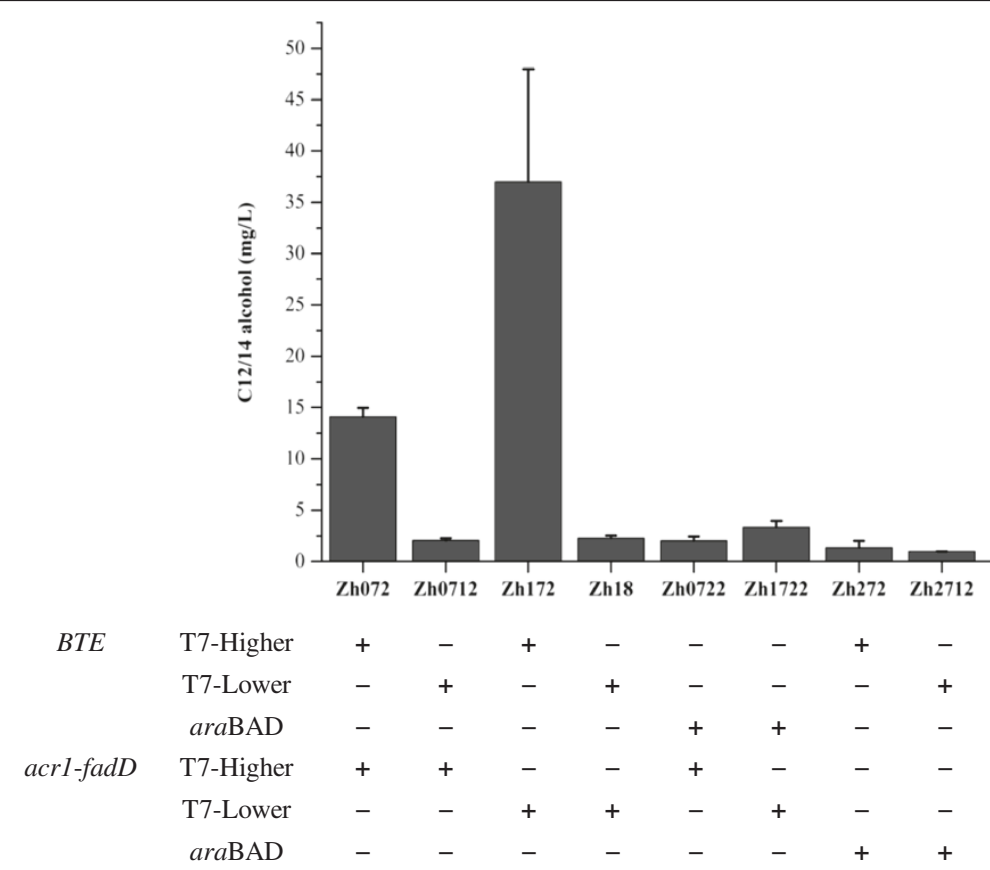

Figure 5 Optimization of the expression level of C12/14 alcohol biosynthesis pathway. Plasmids with different copy numbers and promoters are used to optimize the expression level of C12/14 alcohol biosynthesis pathway. Zh172, with BTE and fadD-acr being carried by a higher copy number plasmid (T7 promoter) and a lower copy number plasmid (T7 promoter), respectively, achieved the highest C12/14 alcohol titer. Zh072 using two higher copy number plasmids (T7 promoter) accumulated a medium amount of C12/14 alcohol. Other strains produced a low concentration of C12/14 alcohol. The error bars represent the range from two independent experiments. 


\section{Fed-batch fermention}

To evaluate the fatty alcohol production in a scaleable process, fed-batch fermentations of Zh172, Zh072 and Zh054 were carried out at 5-L scale. Over the course of the fermentation, Zh172 produced $598.6 \mathrm{mg} / \mathrm{L}$ fatty alcohols, with C12/14 alcohol accounting for $75.0 \%$ (449.2 mg/L), while Zh054 accumulated $101.5 \mathrm{mg} /$ L C16/18 alcohol. The C12/14 alcohol titer of Zh172 was 2.1 folds higher than that of Zh072 (210.1 mg/L) (Figure 6). For Zh072, the C12/14 alcohol productivity was relatively constant at $15.6 \mathrm{mg} / \mathrm{L} / \mathrm{h}$ just after the protein production was triggered with IPTG. However, the linear C12/14 alcohol productivity of Zh172 and C16/18 alcohol productivity of Zh054 appeared approximately $4 \mathrm{~h}$ post-induction, constant at $35.7 \mathrm{mg} /$ $\mathrm{L} / \mathrm{h}$ and $6.5 \mathrm{mg} / \mathrm{L} / \mathrm{h}$, respectively. In addition, a maximal $\mathrm{C} 12 / 14$ alcohol yield of $1.0 \%$ (i.e. $0.01 \mathrm{gC} 12 / 14$ alcohol/g carbon source consumed) and a maximal C16/18 alcohol yield of $0.23 \%$ were observed during the post-induction, linear fatty alcohol production period (Figure 6).

It was worth noting that, at the end of the fermentation, 621.1 $\mathrm{mg} / \mathrm{L} \mathrm{C16/18} \mathrm{FFA} \mathrm{and} \mathrm{133.4} \mathrm{mg/L} \mathrm{C12/14}$ FFA were unutilized by Zh054 and Zh072, respectively. Contrastively, only $316.0 \mathrm{mg} / \mathrm{L}$ and $39.4 \mathrm{mg} / \mathrm{L}$ corresponding FFAs were unutilized by Zh054 and Zh072 at the 8-h time point, when both Zh054 and Zh072 produced fatty alcohols at a maximum productivity. It demonstrated that the produced FFAs were no longer converted to fatty alcohols in the later stage of the fermentation. The fatty alcohol production ceased ahead of the production of FFAs.

\section{Discussion}

Expression of alcohol-forming fatty acyl-CoA reductases in $E$. coli can result in the biosynthesis of fatty alcohols from endogenous $E$. coli fatty acids, but the levels were quite low [19]. To improve the production of fatty alcohols, Steen et al. carried out a further genetic modification of $E$. coli, and achieved an increased titer $(\sim 60 \mathrm{mg} / \mathrm{L})$ of the medium chain fatty alcohols (C12 or C14 alcohols) [3]. In their strategy, they employed thioesterases with different substrate specificities to tailor the composition of the FFAs, and used the aldehydeforming fatty acyl-CoA reductase Acr1 for the conversion of fatty acyl-CoAs to fatty aldehydes. The synthesized fatty aldehydes can be further converted to fatty alcohols by an unknown alcohol dehydrogenase/aldehyde reductase of E. coli [6].

However, Steen et al. just obtained a small quantity of C16/18 alcohol, though they used the thioesterase 'TesA, which was capable of yielding a large proportion of C16/18 FFA [3]. As aforementioned, expression of Acr1 in $E$. coli can only obtain a predominant C12/14 alcohol, even if the longer FFAs are supplied. Therefore, their poor C16/18 alcohol production is probably attributed to the Acr1 they employed. However, Reiser and Somerville spectroscopically assayed the substrate specificity of an unpurified Acr1 protein by measuring the acylCoA-dependent oxidation of NADPH, and found Acr1 had
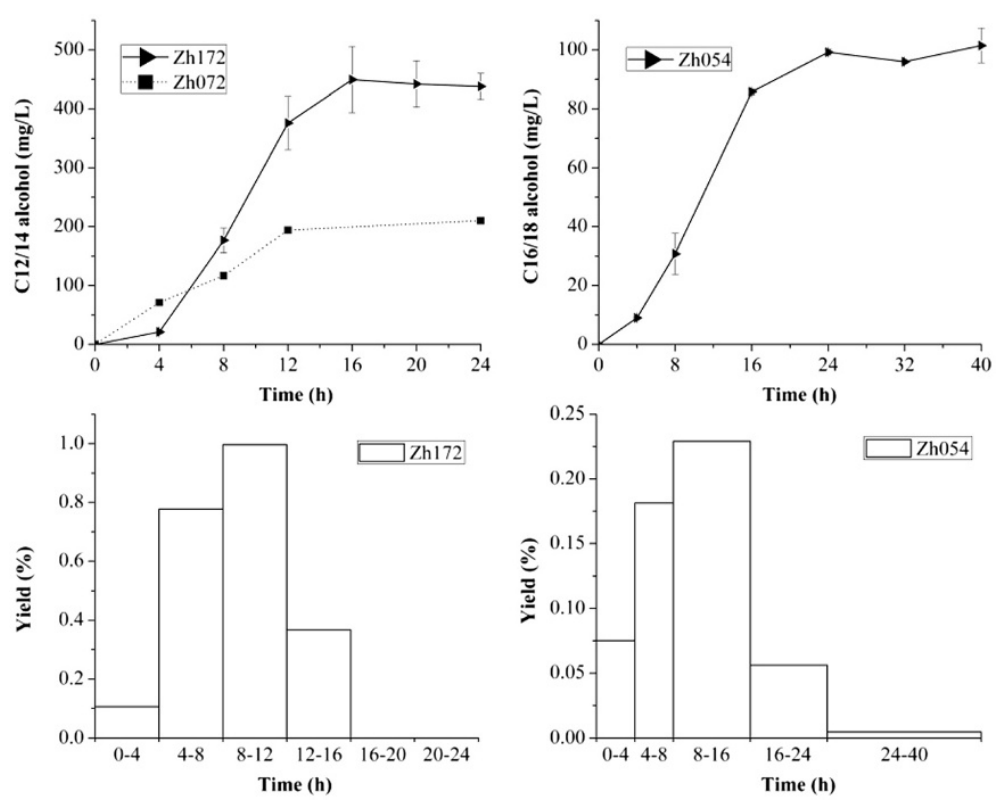

Figure 6 Characterization of fatty alcohol production of Zh172, Zh072 and Zh054 in the fed-batch fermentation. A, B, the production of C12/14 alcohol (A) and C16/18 alcohol (B) in fed-batch fermentations; C, D, the yield (g product/100 g glucose) of Zh172 (C) and Zh054 (D). The error bars represent the range from two independent experiments. 
the biggest substrate preference towards C16/18 acyl-CoAs [6]. It suggests that the endogenous alcohol dehydrogenase/aldehyde reductase of $E$. coli may have substrate preference for fatty aldehydes with shorter chain lengths, and thus block the conversion of C16/18 aldehydes to corresponding alcohols. This bioconversion process will be much clearer if the purified Acr1 protein can be characterized by determining its synthesized products.

We are cognizant of the fact that it is impossible to obtain all our desired fatty alcohols just by tailoring the composition of FFAs. We thus replaced Acr1 with FAR as the fatty acyl-CoA reductase for the production of long chain fatty alcohols, given FAR preferred the longer acyl groups. To data, no research was performed to optimize the long chain fatty alcohol biosynthesis pathway. Therefore, we combined the expression of thioesterase and acyl-CoA synthase with FAR to enhance the long chain fatty alcohol production from glucose. Our achieved titer of $101.5 \mathrm{mg} / \mathrm{L}$ represents the highest C16/18 alcohol production ever reported $[4,19]$.

In addition, Steen et al. found the importance of acylCoA synthase in improving the fatty alcohol production [3]. It is of significance to explore and find a proper acyl-CoA synthase that capable of enhancing the production of C12/14 or C16/18 alcohol. We found FadD possessed broad substrate specificity and high catalytic activity, based on the investigation of three different acyl-CoA synthases from E. coli (FadD), B. subtilis (Yngl) and S. cerevisiae (FAA2), respectively. FadD can convert most of the C12-C18 FFAs into their activated forms fatty acyl-CoAs, and it was suitable for the production of both C12/14 and C16/18 alcohols.

The level of free CoA may also play an important role in the conversion of FFAs to fatty acyl-CoAs, since free CoA directly participates the reaction as a substrate. Once the fatty acyl-CoAs accumulate, that will lead to a reduction of free CoA. The decreased level of free CoA may further block the activation of FFAs. In contrast, increased level of free CoA will benefit the conversion of FFAs to fatty acyl-CoAs.

With a series of combination across different thioesterases, acyl-CoA synthases and fatty acyl-CoA reductases, we constructed two engineered strains (Zh072 and Zh054) that were capable of high-specificity production of C12/14 and C16/18 alcohols, respectively. Lennen et al. achieved a high level of fatty acid production by using a medium-strength $\mathrm{P}_{\mathrm{BAD}}$ [20]. Therefore, it is possible to enhance the fatty alcohol production by optimization of the expression level of fatty alcohol biosynthesis pathway. No enhanced fatty alcohol production was obtained when either BTE or acrl was under the control of $\mathrm{P}_{\mathrm{BAD}}$. However, an obviously improved fatty alcohol production was achieved by optimization of the plasmid copy number. This result provides a useful clue for enhancing the fatty alcohol production. Of course, a more detailed optimization of the expression level is still needed to further improve the production of fatty alcohols.

Given no process data is available on the fatty alcohol production by engineered $E$. coli, we evaluated the performances of three well-performed strains in the fed-batch fermentation. The maximum fatty alcohol productivity was observed in the early stage of the post-induction. Over this phase, fatty alcohol production ceased. Given FFAs can be also converted to fatty aldehydes [6,21-23], maybe the released FFAs were predominately transformed to fatty aldehydes instead of fatty alcohols in the latter stage. To make the fermentation processes be clearer, it is needed to perform some further investigations focusing on the production of fatty aldehydes.

The lower titer in our shake-flask study was probably attributed to the low buffering capacity of our culture medium, whose $\mathrm{pH}$ decreased rapidly with the growth of cells. The resultant lower biomass caused the decreased fatty alcohol production. The fatty alcohol production dramatically increased in the fed-batch fermentation with $\mathrm{pH}$ adaption.

In addition, given that fatty acyl-CoA reductases need the participation of coenzyme NADPH [17], the production of fatty alcohols may be enhanced by expressing the NADPH regeneration system such as phosphite dehydrogenase and glucose-6-phosphate dehydrogenase in E. coli.

\section{Conclusions}

Given the importance of the $\mathrm{C} 12-\mathrm{C} 18$ fatty alcohols as surfactants and the issues associated with petroleum and natural oil feedstocks, increasing attention is being paid to seeking after the low-cost renewable routes from sugars. In addition, it will have a better downstream application to obtain the fatty alcohol products with narrow-range distribution of carbon chain lengths. Therefore, the ultimate objective of this study was to realize the high-level and selective production of C12/14 and C16/18 alcohols in engineered E. coli by optimization of the fatty alcohol biosynthesis pathway. To our knowledge, this is the first report on selective production of $\mathrm{C} 12 / 14$ and C16/18 alcohols in engineered microbes. High-specificity production of both $\mathrm{C} 12 / 14$ and $\mathrm{C} 16 / 18$ alcohols has been obtained. The C12/14 alcohol and C16/18 alcohol occupied $75.0 \%$ and $89.2 \%$ of total fatty alcohols produced by Zh172 and Zh054, respectively. Without detailed optimization of the production conditions, an encouraging $598.6 \mathrm{mg} / \mathrm{L}$ of fatty alcohols was finally achieved, representing the highest fatty alcohol production achieved so far. In addition, the $101.5 \mathrm{mg} / \mathrm{L}$ 
89.2\% C16/18 alcohol suggests an important breakthrough in C16/18 alcohol production. A more detailed optimization of the expression level of fatty alcohol biosynthesis pathway may contribute to a further improvement of fatty alcohol production. Our study provides the groundwork for microbial production of a surfactant range of fatty alcohols from the rich lignocellulose sources.

\section{Methods}

\section{Bacterial strains, media, and growth conditions}

The bacterial strains used in this study are listed in Table 1. E. coli BL21(DE3) (Invitrogen, Carlsbad, CA) was used as the host to overproduce proteins. During strain construction, cultures were grown aerobically at $37^{\circ} \mathrm{C}$ in Luria Broth $(10 \mathrm{~g} / \mathrm{L}$ tryptone, $10 \mathrm{~g} / \mathrm{L} \mathrm{NaCl}$, and $5 \mathrm{~g} / \mathrm{L}$ yeast extract). Kanamycin $(50 \mathrm{mg} / \mathrm{L})$ or chloramphenicol $(34 \mathrm{mg} / \mathrm{L})$ was added if necessary. For initial production experiments in shake flasks, strains were grown in a M9 medium (15.13 g/L Na $2 \mathrm{HPO}_{4} \cdot 12 \mathrm{H}_{2} \mathrm{O}$, $3 \mathrm{~g} / \mathrm{L} \quad \mathrm{KH}_{2} \mathrm{PO}_{4}, 1 \mathrm{~g} / \mathrm{L} \quad \mathrm{NH}_{4} \mathrm{Cl}, 0.5 \mathrm{~g} / \mathrm{L} \mathrm{NaCl}, 2 \mathrm{mM}$ $\mathrm{MgSO} 4)$ containing $20 \mathrm{~g} / \mathrm{L}$ of glucose or $20 \mathrm{~g} / \mathrm{L}$ of glycerol. The engineered strains were fed with glycerol as carbon source if they carried the recombinant plasmids using araBAD promoter, otherwise they were fed with glucose. Another $0.9 \%$ beef extract was added to the M9 medium for the conversion of exogenous fatty acids to fatty alcohols. Protein production was induced with $0.5 \mathrm{mM}$ isopropyl $\beta$-D-thiogalactoside (IPTG) at $30^{\circ} \mathrm{C}$. For the fed-batch fermentation in a $5 \mathrm{~L} \mathrm{BIOSTAT}^{\circledR} \mathrm{B}$ plus fermentor (Sartorius Stedim Biotech $\mathrm{GmbH}$, Goettingen, Germany), strains were grown in the defined batch medium consisted of the following: $7.5 \mathrm{~g} / \mathrm{L}$ $\mathrm{K}_{2} \mathrm{HPO}_{4} \cdot 3 \mathrm{H}_{2} \mathrm{O}, 2.1 \mathrm{~g} / \mathrm{L}$ citric acid monohydrate, $0.3 \mathrm{~g} / \mathrm{L}$ ferric ammonium citrate, $2.92 \mathrm{~g} / \mathrm{L}(\mathrm{NH} 4)_{2} \mathrm{SO}_{4}, 2 \mathrm{mM}$ $\mathrm{MgSO}_{4}$, trace metals mix $\left(2.86 \mathrm{mg} / \mathrm{L} \mathrm{H}_{3} \mathrm{BO}_{3}, 1.81 \mathrm{mg} / \mathrm{L}\right.$ $\mathrm{MnCl}_{2} \cdot 4 \mathrm{H}_{2} \mathrm{O}, 0.222 \mathrm{mg} / \mathrm{L} \quad \mathrm{ZnSO}_{4} \cdot 7 \mathrm{H}_{2} \mathrm{O}, 0.39 \mathrm{mg} / \mathrm{L}$ $\mathrm{Na}_{2} \mathrm{MoO}_{4} \cdot 2 \mathrm{H}_{2} \mathrm{O}, 0.079 \mathrm{mg} / \mathrm{L} \quad \mathrm{CuSO}_{4} \cdot 5 \mathrm{H}_{2} \mathrm{O}, 49.4 \mu \mathrm{g} / \mathrm{L}$ $\left.\mathrm{Co}\left(\mathrm{NO}_{3}\right)_{2} \cdot 6 \mathrm{H}_{2} \mathrm{O}\right)$. The fermentation temperature was controlled at $30^{\circ} \mathrm{C}$ and the $\mathrm{pH}$ at 7.0. The $\mathrm{pH}$ was maintained using $\mathrm{NH}_{3} \cdot \mathrm{H}_{2} \mathrm{O}$. Cells were induced at an $\mathrm{OD}_{600}$ of $\sim 15$ using $0.5 \mathrm{mM}$ IPTG. The glucose feed solution was continuously added into the cultures at the rate of $3 \sim 5 \mathrm{~g} / \mathrm{L} / \mathrm{h}$, and the residual glucose in the cultures was maintained at $0.1 \sim 0.3 \mathrm{~g} / \mathrm{L}$.

\section{Regents}

The arachidyl alcohol and arachidic acid were ordered from Acros Organics (Geel, Belgium) and Alfa Aesar (Ward Hill, MA), respectively. All restriction enzymes and T4 DNA ligatase were purchased from Fermentas (Vilnius, Lithuania). The Pyrobest DNA polymerase was supplied by Takara Biotechnology (Dalian, China). The pACYCDuet-1, pCOLADuet-1 and pET-30a(+) expression vectors were purchased from Novagen (Darmstadt, Germany). Oligonucleotides were ordered from BGI (Beijing, China).

\section{Plasmid construction}

Genes derived from E. coli str. K12 substr. MG1655 (fadD [Genbank: 946327], and 'tes $A$, a leaderless version of tesA [Genbank: 945127]) [24,25], Saccharomyces cerevisiae S288c (FAA2 [Genbank: NM_001178906]), Bacillus subtilis ATCC 23857 (yngl [Genbank: 939955]), and Acinetobacter baylyi ATCC 33305 (acr1 [Genbank: U77680]) were obtained by polymerase chain reaction (PCR) using Pyrobest DNA polymerase. The CER4 gene [Genbank: NM_119537] was amplified by PCR using the total cDNA of Arabidopsis thaliana as the template. The BTE gene [Genbank: M94159] of Umbellularia californica and a codon-optimized FAR gene of Simmondsia chinensis [Genbank: JQ768345] were chemically synthesized. Custom oligonucleotides (primers) for all PCR amplifications are shown in Table 2.

The amplified FAR gene was firstly gel purified, then digested with $\mathrm{NcoI}$ and BamHI, and finally ligated into pACYCDuet-1 that had been digested with the same restriction enzymes, yielding pZh01. In the same means, the BglII-XhoI digested $f a d D$, NdeI-EcoRV digested $F A A 2$ and EcoRV-XhoI digested yngl were ligated into pZh01 to yield pZh05, pZh09 and pZh10, respectively. For the construction of $\mathrm{pZh07}$ and pZh08, the FAR located at pZh05 was replaced with acr 1 and CER4, respectively. To yield pZh11 and pZh12, the acr1 gene was removed from the plasmid pZh07 by restriction enzyme digestion with NcoI/EcoRI, gel purified, and ligated into pZh09 and pZh10 whose FAR gene had been removed by restriction enzyme digestion. To create pZh17 and pZh27, acr1-fadD, cut from pZh07 with $\mathrm{NcoI} / \mathrm{XhoI}$, was cloned into corresponding sites of pCOLADuet-1 and pBAD/Myc-HisA, respectively. Three sets of primers, BTE-NdeF/BTE-NotR, BTE-NdeF/BTEXhoR and BTE-NcoF/BTE-XhoR, were used to amplify the thioesterase gene BTE. The amplified BTEs were inserted into the NdeI/NotI site of pET-30a(+), NdeI/ XhoI site of pCOLADuet-1 and pACYCDuet-1, and $\mathrm{NcoI} / \mathrm{XhoI}$ site of $\mathrm{pBAD} / \mathrm{Myc}$-HisA, respectively. The resulted recombinant plasmids were designated as pYN2 [26], pYN12, pXW2 and pYN22, respectively. A primer set of T7min-BamF/BTE-NotR was used to amplify the $B T E$ with a T7 promoter (T7BTE) using pYN2 as the template, the PCR product was digested with BamHI/ NotI, and then inserted into pZh17 to yield pZh18. As for the thioesterases gene 'tes $A$, the NcoI/EcoRI digested 'tes $A$ were ligated into corresponding sites of pCOLADuet-1, creating pLL4. Plasmids used in this work are listed in Table 1. 
Table 1 Bacterial strains and plasmids used in this study

\begin{tabular}{|c|c|c|}
\hline Plasmid or strain & Relevant genotype & Reference or source \\
\hline \multicolumn{3}{|l|}{ Plasmids } \\
\hline pET-30a(+) & pBR322 ori lacl T7lac Kan ${ }^{r}$ & Novagen \\
\hline pACYCDuet-1 & P15A ori lacl T7lac $\mathrm{Cm}^{r}$ & Novagen \\
\hline pCOLADuet-1 & ColA ori lacl T7lac Kan ${ }^{r}$ & Novagen \\
\hline $\mathrm{pBAD} / \mathrm{Myc}-\mathrm{HisA}$ & pBR322 ori araBAD Amp ${ }^{r}$ & Invitrogen \\
\hline pZh05 & pACYCDuet-1 harboring FAR from Jojoba and fadD from E. coli & This study \\
\hline pZh07 & pACYCDuet-1 harboring acr 1 from A. baylyi and fadD from E. coli & This study \\
\hline pZh08 & pACYCDuet-1 harboring CER4 from A. thaliana and fadD from E. coli & This study \\
\hline pZh09 & pACYCDuet-1 harboring FAR from Jojoba and yngl from B. subtilis & This study \\
\hline pZh10 & pACYCDuet-1 harboring FAR from Jojoba and FAA2 from S. cerevisiae & This study \\
\hline pZh11 & pACYCDuet-1 harboring acr1 from A. baylyi and $y n g /$ from B. subtilis & This study \\
\hline pZh12 & pACYCDuet-1 harboring acr1 from A. baylyi and FAA2 from S. cerevisiae & This study \\
\hline pZh17 & pCOLADuet-1 harboring acr 1 from A. baylyi and fadD from E. coli & This study \\
\hline pZh18 & pCOLADuet-1 harboring acr 1 from A. baylyi, fadD from E. coli and BTE from U. californica & This study \\
\hline pZh27 & pBAD/Myc-HisA harboring acrl from A. baylyi and fadD from E. coli & This study \\
\hline pYN2 & pET-30a(+) harboring BTE from U. californica & 26 \\
\hline pLL4 & pCOLADuet-1 harboring 'tesA from E. coli & This study \\
\hline pYN12 & pCOLADuet-1 harboring BTE from U. californica & This study \\
\hline pXW2 & pACYCDuet-1 harboring BTE from U. californica & This study \\
\hline pYN22 & pBAD/Myc-HisA harboring BTE from U. californica & This study \\
\hline \multicolumn{3}{|l|}{ Strains } \\
\hline $\mathrm{DH} 5 \mathrm{a}$ & $\begin{array}{l}F^{-}, \varphi 80 \mathrm{~d} / a c Z \Delta \mathrm{M} 15, \Delta\left(\text { lacZYA-argF)U169, deoR,recA1, endA1, hsdR17(rk',mk }{ }^{+}\right) \text {, phoA, } \\
\text { supE44, } \lambda \text {-,thi'1, gyrA96, relA1 }\end{array}$ & Takara \\
\hline BL21 (DE3) & $\mathrm{F}^{-}$ompT gal dem lon hsdSB(r- $\left.\mathrm{r}_{B}^{-}\right) \lambda(\mathrm{DE} 3)$ & Invitrogen \\
\hline Zh05 & E. coli BL21 (DE3) bearing pZh05 & This study \\
\hline Zh07 & E. coli BL21 (DE3) bearing pZh07 & This study \\
\hline Zh08 & E. coli BL21 (DE3) bearing pZh08 & This study \\
\hline Zh072 & E. coli BL21 (DE3) bearing pZh07 and pYN2 & This study \\
\hline Zh112 & E. coli BL21 (DE3) bearing pZh11 and pYN2 & This study \\
\hline Zh122 & E. coli BL21 (DE3) bearing pZh12 and pYN2 & This study \\
\hline Zh054 & E. coli BL21 (DE3) bearing pZh05 and pLL4 & This study \\
\hline Zh094 & E. coli BL21 (DE3) bearing pZh09 and pLL4 & This study \\
\hline Zh104 & E. coli BL21 (DE3) bearing pZh10 and pLL4 & This study \\
\hline Zh0712 & E. coli BL21 (DE3) bearing pZh07 and pYN12 & This study \\
\hline Zh172 & E. coli BL21 (DE3) bearing pZh17 and pXW2 & This study \\
\hline Zh18 & E. coli BL21 (DE3) bearing pZh18 & This study \\
\hline Zh0722 & E. coli BL21 (DE3) bearing pZh07 and pYN22 & This study \\
\hline Zh1722 & E. coli BL21 (DE3) bearing pZh17 and pYN22 & This study \\
\hline Zh272 & E. coli BL21 (DE3) bearing pZh27 and pXW2 & This study \\
\hline Zh2712 & E. coli BL21 (DE3) bearing pZh27 and pYN12 & This study \\
\hline
\end{tabular}

\section{Detection of metabolites}

The harvested cultures were first heated with boiled water for 20 minutes to partly lyse the cells. Then the $\mathrm{pH}$ values of the cultures were adjusted to 2.0 using hydrochloric acid. Next chloroform-methanol (v/v, 2:1) was added into the cultures. The resultant blends were vortexed for a few minutes and then left overnight to extract the fatty alcohols and fatty acids. The arachidyl 
Table 2 Primers used in this study

\begin{tabular}{|c|c|c|}
\hline Gene & Primer name & Sequence $\left(5^{\prime} \rightarrow 3^{\prime}\right)$ \\
\hline \multirow[t]{5}{*}{ BTE } & BTE-NdeF & GGAATTCCATATGGCCACCACCTCTTTAG \\
\hline & BTE-NotR & ATAAGAATGCGGCCGCTTACACCCTCGGTTCTGCG \\
\hline & BTE-NcoF & CTAGCCATGGCCACCACCTCTTTAGC \\
\hline & BTE-XhoR & CACCTCGAGTTACACCCTCGGTTCTGCG \\
\hline & T7min-BamF & CAGGGATCCTAATACGACTCACTATAGG \\
\hline \multirow[t]{2}{*}{ 'tes $A$} & tesA-NcoF & CATGCCATGGCGGACACGTTATTGATTCTG \\
\hline & tesA-EcoR & CAGGAATTCTTATGAGTCATGATTTACTAAAG \\
\hline \multirow[t]{2}{*}{ fadD } & fadD-BglF & CTCAGATCTCATGAAGAAGGTITGGCTTAACC \\
\hline & fadD-XhoR & CACCTCGAGTTATCAGGCTITATTGTCCACTITG \\
\hline \multirow[t]{2}{*}{ yngl } & yngl-Eco32F & CACGATATCGATGGCTGAACTCATCCATTCC \\
\hline & yngl-XhoR & CACCTCGAGTCATTGACATGATGATAAGTTG \\
\hline \multirow[t]{2}{*}{ FAA2 } & FAA2-NdeF & GGAATTCCATATGGCCGCTCCAGATTATGCAC \\
\hline & FAA2-Eco32R & CACGATATCTTACTAAAGCTTTTCTGTCTTGACTAG \\
\hline \multirow[t]{2}{*}{$F A R$} & FAR-NcoF & CATGCCATGGAGGAAATGGGCAGC \\
\hline & FAR-BamR & CGGGATCCTTAGTTAAGAACGTGCTCCAC \\
\hline \multirow[t]{2}{*}{ CER4 } & CER4-NcoF & CATGCCATGGGCATGTCGACAGAAATGGAGGTCG \\
\hline & CER4-BamR & CGGGATCCTTAGAAGACATACTTAAGCAGCCC \\
\hline \multirow[t]{2}{*}{ acr1 } & acr1-NcoF & CATGCCATGGTGAACAAAAAACTTGAAGC \\
\hline & acr1-BamR & CGGGATCCTTACCAGTGTTCGCCTG \\
\hline
\end{tabular}

Underlines indicate restriction enzyme sites.

alcohol and arachic acid were added into the cultures and used as the internal standards for the analysis of fatty alcohols and fatty acids, respectively. The desired products of the shake-flask culture were quantified by gas chromatography-mass spectrometry (GC-MS) using an Agilent 7890A system equipped with a HP-INNOWax (30 m $\times 0.25 \mathrm{~mm} ; 0.25 \mu \mathrm{m}$ film thickness). Helium was used as the carrier gas. The injection port was held at $250^{\circ} \mathrm{C}$, and the following oven temperature program was carried out: $100^{\circ} \mathrm{C}$ for $2 \mathrm{~min}$, increase of $10^{\circ} \mathrm{C} / \mathrm{min}$ to $240^{\circ} \mathrm{C}$ for $15 \mathrm{~min}$. The desired products of the fermentor culture were quantified by a gas chromatograph (GC) equipped with a flame ionization detector. The separation of FFAs was performed using a CP-FFAP CB capillary column $(25 \mathrm{~m} \times 0.25 \mathrm{~mm}$; $0.2 \mu \mathrm{m}$ film thickness) purchased from Agilent Technologies (Santa Clara, CA). The oven temperature was initially held at $100^{\circ} \mathrm{C}$ for $1 \mathrm{~min}$, then raised with a gradient of $10^{\circ} \mathrm{C} / \mathrm{min}$ until reaching $250^{\circ} \mathrm{C}$, and finally held for $10 \mathrm{~min}$. Nitrogen was used as the carrier gas. The injector and detector were held at $270^{\circ} \mathrm{C}$ and $300^{\circ} \mathrm{C}$, respectively.

\section{Abbreviations}

ACP: Acyl carrier protein; CoA: Coenzyme A; FFA: Free fatty acid; IPTG: Isopropyl $\beta$-D-thiogalactoside; PCR: Polymerase chain reaction; GC: Gas chromatography; GC-MS: Gas chromatography-mass spectrometry.

\section{Competing interests}

The authors declare that they have no competing interests.

\section{Acknowledgements}

This work was financially supported by Guangdong Province-Chinese Academy of Sciences Joint Project (2009B091300146), National Natural Science Foundation of China (21106170), and Main Direction Program of Knowledge Innovation of Chinese Academy of Sciences (KSCX2-EW-G-13). We also thank Dr. Wenna Guan and Cong Wang for assistance with the GC- MS analysis.

\section{Author details}

${ }^{1}$ Qingdao Institute of Bioenergy and Bioprocess Technology, Chinese Academy of Sciences, No.189 Songling Road, Qingdao Laoshan District 266101, China. ${ }^{2}$ Graduate University of Chinese Academy of Sciences, Beijing 100049, China. ${ }^{3}$ College of Food Science, Sichuan Agricultural University, Yaan 625014, China.

\section{Authors' contributions}

MX and GZ developed the idea for the study. $Y Z$ designed the research. $Y Z$ did the literature review and prepared the manuscript. $M X$ and $G Z$ helped to revise the manuscript. $Y Z$ and $L L$ did the majority of the lab work, plasmid construction, strain cultivation and product detection. QL participated in the plasmid construction and product detection. $Y Z$, JY and $H L$ performed the fed-batch fermentation. JY, XW, WL and XX participated in the product detection. All authors read and approved the final manuscript.

Received: 15 December 2011 Accepted: 20 May 2012

Published: 20 May 2012

\section{References}

1. Hreczuch W, Szymanowski J: Synthesis of surfactants with narrow-range distribution of the polyoxyethylene chain. J Am Oil Chem Soc 1996, 73:73-78.

2. Hatcher D: Fatty alcohol sulfates. J Am Oil Chem Soc 1957, 34:175-178.

3. Steen EJ, Kang Y, Bokinsky G, Hu Z, Schirmer A, McClure A, del Cardayre SB, Keasling JD: Microbial production of fatty-acid-derived fuels and chemicals from plant biomass. Nature 2010, 463:559-562.

4. Tan X, Yao L, Gao Q, Wang W, Qi F, Lu X: Photosynthesis driven conversion of carbon dioxide to fatty alcohols and hydrocarbons in cyanobacteria. Metab Eng 2011, 13:169-176.

5. Lu X, Vora H, Khosla C: Overproduction of free fatty acids in E. coli: Implications for biodiesel production. Metab Eng 2008, 10:333-339.

6. Reiser S, Somerville C: Isolation of mutants of Acinetobacter calcoaceticus deficient in wax ester synthesis and complementation of one mutation with a gene encoding a fatty acyl coenzyme A reductase. J Bacteriol 1997, 179:2969-2975.

7. Vioque J, Kolattukudy PE: Resolution and purification of an aldehydegenerating and an alcohol-generating fatty acyl-CoA reductase from pea leaves (Pisum sativum L.). Arch Biochem Biophys 1997, 340:64-72.

8. Liu X, Sheng J, Curtiss R: Fatty acid production in genetically modified cyanobacteria. Proc Natl Acad Sci 2011, 108:6899-6904.

9. Voelker TA, Davies HM: Alteration of the specificity and regulation of fatty acid synthesis of Escherichia coli by expression of a plant medium-chain acyl-acyl carrier protein thioesterase. J Bacteriol 1994, 176:7320-7327.

10. Yuan L, Voelker TA, Hawkins DJ: Modification of the substrate specificity of an acyl-acyl carrier protein thioesterase by protein engineering. Proc Natl Acad Sci 1995, 92:10639-10643.

11. Voelker TA, Worrell AC, Anderson L, Bleibaum J, Fan C, Hawkins DJ, Radke SE, Davies HM: Fatty acid biosynthesis redirected to medium chains in transgenic oilseed plants. Science 1992, 257:72-74.

12. Bonner WM, Bloch K: Purification and properties of fatty acyl thioesterase I from Escherichia coli. J Biol Chem 1972, 247:3128-3133.

13. Morgan-Kiss RM, Cronan JE: The Escherichia coli fadK (ydiD) gene encodes an anerobically regulated short chain acyl-CoA synthetase. J Biol Chem 2004, 279:37324-37333.

14. Hettema Ewald H, van Roermund Carlo WT, Ben Distel, van den Berg Marlene, Cristina Vilela, Claudina Rodrigues-Pousada, Wanders RJA, Tabak HF: The $A B C$ transporter proteins Pati and Pat2 are required for import of long-chain fatty acids into peroxisomes of Saccharomyces cerevisiae. EMBO J 1996, 15:3813-3822.

15. Rowland O, Zheng H, Hepworth SR, Lam P, Jetter R, Kunst L: CER4 encodes an alcohol-forming fatty acyl-coenzyme $A$ reductase involved in cuticular wax production in Arabidopsis. Plant Physiol 2006, 142:866-877. 
16. Kalscheuer R, Stöveken T, Luftmann H, Malkus U, Reichelt R, Steinbüchel A: Neutral lipid biosynthesis in engineered Escherichia coli: jojoba oil-like wax esters and fatty acid butyl esters. Appl Environ Microbiol 2006, 72:1373-1379.

17. Metz JG, Pollard MR, Anderson L, Hayes TR, Lassner MW: Purification of a jojoba embryo fatty acyl-coenzyme A reductase and expression of its CDNA in high erucic acid rapeseed. Plant Physiol 2000, 122:635-644.

18. Santala S, Efimova E, Kivinen V, Larjo A, Aho T, Karp M, Santala V: Improved triacylglycerol production in Acinetobacter baylyi ADP1 by metabolic engineering. Microb Cell Fact 2011, 10:36.

19. Doan TTP, Carlsson AS, Hamberg M, Bülow L, Stymne S, Olsson P: Functional expression of five Arabidopsis fatty acyl-CoA reductase genes in Escherichia coli. J Plant Physiol 2009, 166:787-796.

20. Lennen RM, Braden DJ, West RM, Dumesic JA, Pfleger BF: A process for microbial hydrocarbon synthesis: Overproduction of fatty acids in Escherichia coli and catalytic conversion to alkanes. Biotechnol Bioeng 2010, 106:193-202

21. Ishige T, Tani A, Takabe K, Kawasaki K, Sakai Y, Kato N: Wax ester production from $\mathrm{n}$-alkanes by Acinetobacter $s p$. strain $\mathrm{M}-1$ : ultrastructure of cellular inclusions and role of acyl coenzyme A reductase. Appl Environ Microbiol 2002, 68:1192-1195.

22. Dellomonaco C, Fava F, Gonzalez R: The path to next generation biofuels: successes and challenges in the era of synthetic biology. Microb Cell Fact 2010, 9:3.

23. Santala S, Efimova E, Karp M, Santala V: Real-Time monitoring of intracellular wax ester metabolism. Microb Cell Fact 2011, 10:75.

24. Hyeseon Cho, John E, Cronan J: Escherichia coli thioesterase I, molecular cloning and sequencing of the structural gene and identification as a periplasmic enzyme. J Biol Chem 1993, 268:9238-9245.

25. Cho H, Cronan JE: Defective export of a periplasmic enzyme disrupts regulation of fatty acid synthesis. J Biol Chem 1995, 270:4216-4219.

26. Wang X, Li L, Zheng Y, Zou H, Cao Y, Liu H, Liu W, Xian M: Biosynthesis of long chain hydroxyfatty acids from glucose by engineered Escherichia coli. Bioresour Technol 2012. doi:10.1016/j.biortech.2012.02.119.

doi:10.1186/1475-2859-11-65

Cite this article as: Zheng et al:: Optimization of fatty alcohol

biosynthesis pathway for selectively enhanced production of $\mathrm{C} 12 / 14$ and C16/18 fatty alcohols in engineered Escherichia coli. Microbial Cell Factories 2012 11:65.

\section{Submit your next manuscript to BioMed Central and take full advantage of:}

- Convenient online submission

- Thorough peer review

- No space constraints or color figure charges

- Immediate publication on acceptance

- Inclusion in PubMed, CAS, Scopus and Google Scholar

- Research which is freely available for redistribution 